\title{
A new part consolidation method to embrace the design freedom of additive manufacturing
}

\author{
Sheng Yang ${ }^{1}$, Yunlong Tang ${ }^{1}$, and Yaoyao Fiona Zhao ${ }^{1^{*}}$ \\ ${ }^{1}$ McGill University, Montreal, Canada \\ sheng.yang@mail.mcgill.ca,tang.yunlong@mail.mcgill.ca,yaoyao.zhao@mcgill.ca
}

\begin{abstract}
As Additive Manufacturing(AM) evolves from Rapid Prototyping(RP) to the end-of-use product manufacturing process, manufacturing constraints have been largely alleviated and design freedom for part consolidation is extremely broadened. AM enabled part consolidation method promises a more effective way to achieve part count reduction and the ease of assembly compared with traditional Design for Manufacture and Assembly (DFMA) method. However, how to achieve AM enabled part consolidation is not well developed. In this paper, a new part consolidation method comprehensively considering function integration and structure optimization is proposed. This presented method is characterized by two main modules. The first one is to achieve better functionality through surfacelevel function integration and sequential part-level function integration based on design specifications with an initial CAD model which is designed for conventional manufacturing process. The other module is to realize better performance through the introduction and optimization of heterogeneous lattice structures according to performance requirements. The proposed part consolidation method highlights itself from the perspective of functionality achievement and performance improvement. An example of triple clamp is studied to verify the effectiveness of the proposed model. The optimized results show that the part count has been reduced from 19 to 7 with a less weight by $20 \%$ and demonstrates better performance.
\end{abstract}

Keywords: part consolidation, Design for Manufacture and Assembly, lattice structure, Additive Manufacturing, function integration

\section{Introduction}

In order to stay competitive in modern production industry, products should be designed and manufactured within the following two opposite objectives: decreasing time and cost; increasing quality and flexibility. Part count reduction as one of the effective ways to reduce process time and cost has received more and more attention in the past decades. One feasible way to realize part count

\footnotetext{
${ }^{*}$ Corresponding author
} 
reduction is part consolidation which is defined as a process of composites fabrication in which multiple discrete parts are designed and fabricated together into a single part, thus reducing the number of fabricated parts and the need to join those parts together [1]. A reduction in the number of assembly operations can have a tremendous impact on production costs and difficulties of products. Firstly, there is no need for dedicated tooling- for example fixture, and fasteners and potential assembly difficulties like joining method is avoided. Furthermore, it is often possible to design the consolidated parts to perform better than the assemblies. Ultimately, a reduction in part count means that product complexity is reduced from management and production perspectives since fewer parts need to be tracked, sourced, inspected. Part consolidation is intensively studied in conventional Design Theory and Methodology (DTM) such as Design for Assembly (DFA) [2], Design for Disassembly (DFD) [3], and Design for Manufacture and Assembly (DFMA) [4]. The problem is that design freedom of part consolidation is heavily stifled by the requirements of Design for Manufacturing (DFM), which leads to the limited reconstruction by only deleting fasteners and merging existing parts together. Moreover, part consolidation stagnates without taking into consideration of structural optimization of the merged design space to achieve better performance; therefore, a global optimal consolidated structure is not achieved. As AM process evolves from RP to the end-of-use product manufacturing method, manufacturing constraints are largely alleviated and the design freedom is extremely expanded. For example, design limitations by conventional manufacturability such as uniform wall thickness, avoiding sharp corners, and minimising weld lines in injection moulding can be overcome by AM [5].

AM is defined by the American Society for Testing Materials (ASTM) as " a process of joining materials to make object from 3D model data, usually layer upon layer, as opposed to subtractive manufacturing methodologies" [6]. From manufacturability perspective, the benefit by taking AM process(also well known as Rapid Manufacturing(RM) at the beginning) is the ability to virtually manufacture parts of any geometric complexity without tooling, which used to be one of the typical restrictive factors of today's product development [7]. From design perspective, the advantage of AM over conventional subtractive or formative methods is well illustrated by the great design freedom. These design freedoms enabled by AM capabilities are reflected in four categories: shape complexity, hierarchical complexity, material complexity and functional complexity [8]. Therefore, the design freedom for part consolidation and performance improvement is largely expanded. In section 2, a literature review of AM enabled design methods for part consolidation and design methods for performance improvement is given.

\section{Literature review}

There are numerous literatures on adapting AM to do part consolidation [8-12]. The first and wellknown reported part consolidation case using AM capabilities was the aircraft duct redesign case [8] shown in Figure 1. Since the limitation of conventional manufacturing processes, 16 parts are needed to be assembled to accomplish this aircraft duct. After part consolidation process, only one part is needed to be fabricated by AM process. Realizing the opportunities brought by RM, Becker etc.[11] introduced some major design guidelines for rapid manufacturing and applied these guidelines to a case study of a mix device. The optimized part has advantages in reduced part count, less assembly effort and advanced functionality. A similar case is a fluid control valve with 18 components, which is redesigned to be a new one consisting of 8 parts with better performance based on DFMA and RP [9]. An important application in automobile was reported by Hopkins et al.[10] to redesign a door assembly that is composed of 11 pieces for an automotive application subjected to environmental burden and financial profits/costs for end-of-life recycling. It is important to note that although all these reported cases produced new and better design to achieve part consolidation, there is no clear 
design framework to implement the design process and the extent of success depends much on designers' experience and understanding of the functional requirements.

To overcome the drawback of difficulty in determining the real optimized characteristics for a given AM process from an initial CAD model that is designed for traditional fabrication processes, a global approach (shown in Figure 2) aiming at defining part shapes from the manufacturing process and the functional requirements is presented by Ponche et al. [12]. This design method is composed of three steps. The first step is a global analysis which allows the delimitation of the design problem in terms of geometrical dimensions in relation to the dimensional characteristics of the AM process. The second step allows the fulfilment of the dimensional and geometrical specifications in relation to the AM process capability and the finishing process characteristics. Finally, the third step allows the fulfilment of the physical and assembly requirements in relation to the capability of the AM process. The proposed design method is applied to a case of a robot hinge made in stainless steel. Based on the building orientation and mechanical behaviour analysis, the final geometry is given (see in Figure 3). However, functional surfaces and functional volumes are not well defined and the gap between these two functional units is not bridged.

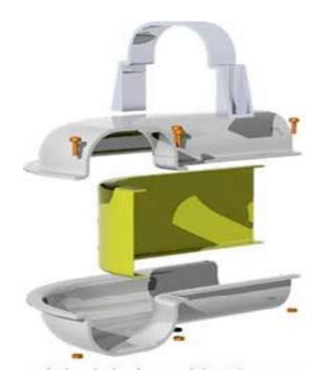

(a) Before consolidation:16 parts

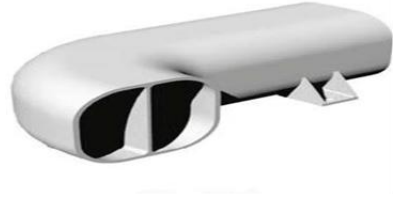

(b) After consolidation: 1 parts

Figure 1: Aircraft duct examples [8]

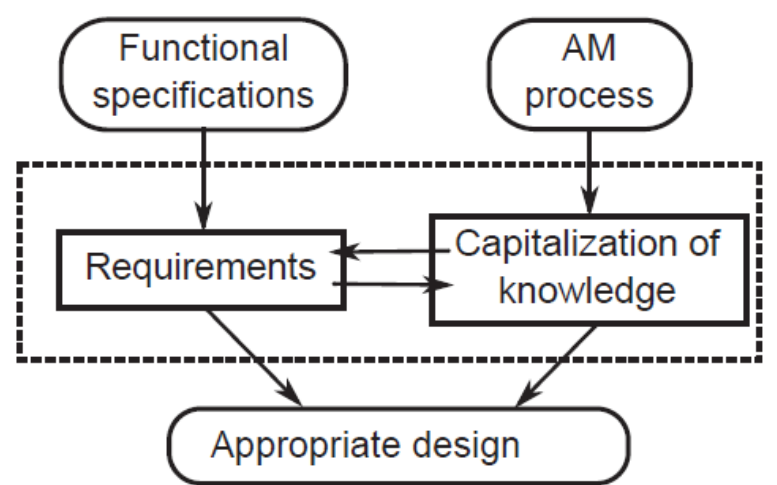

Figure 2: A global design approach [12] 

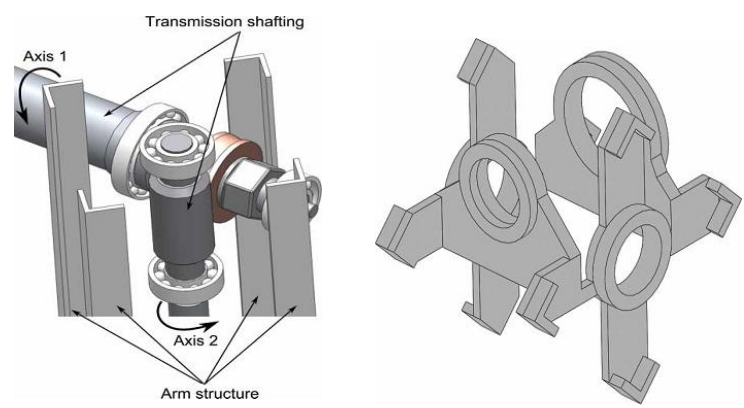

(a) The global view of the studied system (b) The redesigned arm structure

Figure 3: The application of global design approach in a case of robot hinge redesign

Moreover, in the reported related research, structure optimization corresponding to load conditions and other performance requirements are rarely discussed. Actually, the design space derived from part consolidation process can be further partitioned and optimized to achieve better performance. To improve products' performance, lattice structure on a meso-level $(0.1 \sim 10 \mathrm{~mm})$ is widely used [13-15]. This structure is defined as a meso-level structure which consists of an interconnected network of solid struts or plates. By carefully designing the topology of a network of lattice and its struts' thickness, some desired structural properties, such as high stiffness weight ratio and high energy absorption rate, can be achieved. Thus, the lattice structure is usually used to replace the solid material for the further performance improvement.

Design methods for lattice structure can be mainly divided into two types. They are design methods for homogenous lattice and design methods for heterogeneous lattice. For homogenous lattice structure, since the homogenous lattice structure on a meso-level can be regarded as homogenous material, traditional material section method can be used to select an appropriate type of homogenous lattice structure [16]. Compared to design methods for homogenous lattice structure, design methods for heterogeneous lattice are more complex because the struts' thickness, size and orientation of lattice unit cell will unevenly distributed in the design domain. To optimize the struts thickness distribution, some structural optimization methods [17-19] are used. Besides optimizing its struts' thickness distribution, the shape and orientation of lattice unit cell can also be optimized to achieve a better performance $[14,19,20]$. Generally, the heterogeneous lattice structure can always achieve a better performance than its homogenous counterpart [21]. As shown in Figure 4, under the same load condition, compared with the beam with homogenous lattice in (a), the heterogeneous lattice structure in (b) shows a better stiffness since density distribution is in proportion to load condition.

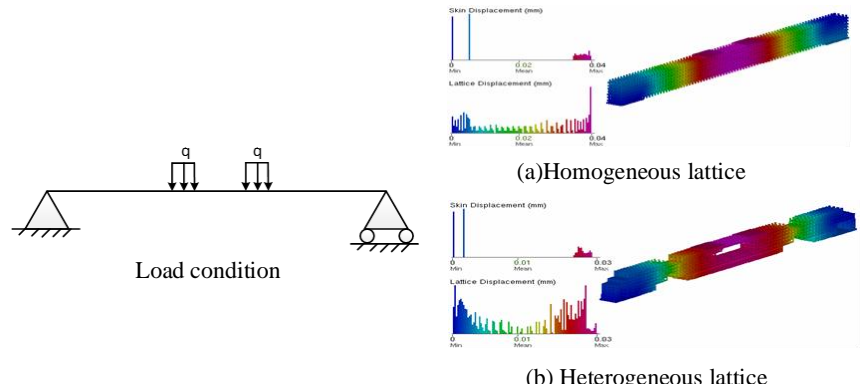

Figure 4: Homogenous lattice and heterogeneous lattice

In order to realize better functionality and better performance with respect to functional requirements and manufacturing constraints, a new part consolidation method which synthesizes function integration and structure optimization is proposed. AM enabled part consolidation is realized 
from a functional standpoint allowing better functional achievement and performance; meanwhile, it increases the scope in the search for better design solutions.

\section{Proposed methodology}

The goal of the presented method is to provide a redesign method that both reduces part count and improves product performance by means of part consolidation at functional level and subsequently through structure optimization. To understand the proposed method, the following terminology must be first defined. Part is defined as a designed object that has no assembly operations in its manufacture. An assembly is a collection of two or more parts. The basic characteristics which the proposed design method should have, and general principles are presented as follows:

- The design method should be suitable for the design with one material type in at least assembly level other than in part level. A design method that starts at part level usually does not have a global view, which is essential to achieve part consolidation.

- The design method should be suitable for the design in multiple design domains, i.e. stiffness, heat and dynamics.

- The design method should include the considerations of design for the ease of process difficulties and for better performance.

- The design method should be a process-based design methodology.

- The design method may include the considerations for cost, time and other aspects like energy consumption to achieve a more cost-effective design.

\subsection{Design framework}

A new part consolidation method which satisfies the basic principles and objectives is shown in Figure 5. In this design method, design specifications are given as the initial inputs, which contains the information of Functional Requirements(FRs), Performance Requirements(PRs) and the initial CAD model designed for conventional manufacturing process. According to Dieter's considerations of a good design [22,23], a function means what it is designed to do, while performance refers to how well the function is achieved. Normally, performance requirements have tolerance while functional requirements do not.

Based on the initial input of this design model, part consolidation process can be divided into two main steps (the highlighted rectangular). The first step is to analyze the initial CAD model and perform part consolidation in functional level according to functional requirements and performance requirements. This step is defined as function integration as shown in Figure 5. In the second step, structure optimization methods are applied to newly generated design space to achieve better performance such as lighter weight, better heat dispatch or dynamic properties under the requirements of performance. The above two steps should also comply with the process constraints of manufacturing, assembly, and standardization. After that, design solutions are verified. If there is no design solution, design flow goes back to function integration and necessary modifications should be done in this step. If design solutions are found, output the design solutions and the original part is redesigned with less part count and better performance. 


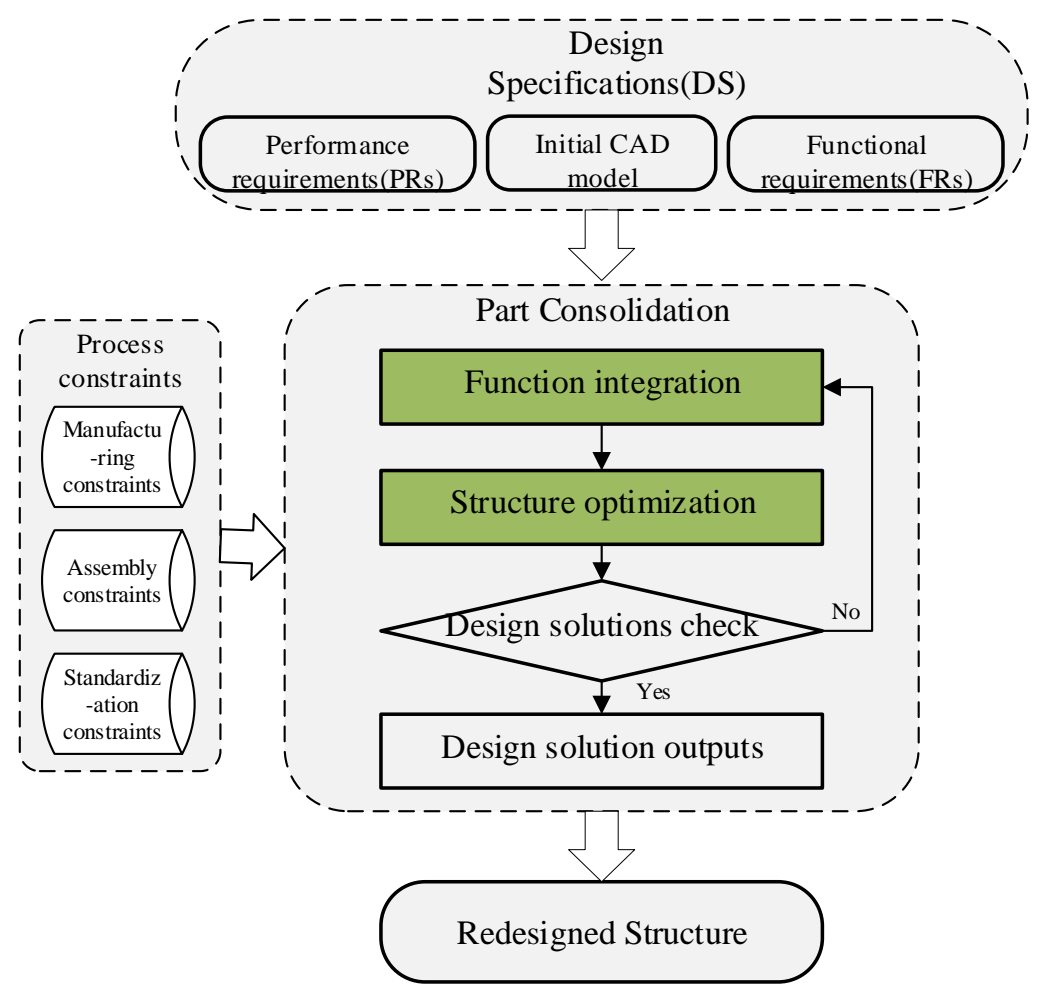

Figure 5: Proposed part consolidation method

\subsection{Function integration}

Generally function integration can be further divided into two main sub-steps: functional surfaces extraction and functional volumes generation. Functional Surface (FS) is defined as a set of surfaces which can fulfill certain FRs. Ideally, if FRs can be decomposed into sub-FRs to the lowest level, one FS is designed to fulfill one corresponding FR. For example, Figure 6 (a) shows a surface of airfoil which can provides lift force (FR) for aircraft. Figure 6 (b) shows that the upper face of the chair is a combination of two FSs to provide support and an aesthetic function(2 FRs) respectively. The interesting part is that the mergence of these two FSs helps to achieve function integration in surface level. Based on FS, Functional Volume (FV) is defined as a geometry volume to link functional surfaces to form a solid to undertake desired performance requirements. As shown in Figure 6 (a), FV is formed by the closed FS. Function volume is an effective way to achieve function integration in part level. Hereby, assume that the element number of FRs set is $\mathrm{X}_{0}$ and the element number of PRs set is $\mathrm{X}_{1}$, so $\mathrm{FS}$ and $\mathrm{FV}$ is represented as follows:

$$
\begin{array}{r}
F S=\left\{f s \mid f s_{i}\left(G, P, A, N, F R_{\alpha}\right)\right\} i=1,2, \cdots, n ; \alpha \in X_{0} \\
F V=\left\{f v \mid f v_{j}\left(\sum_{k=1}^{n_{j}} f s_{k}, P R_{\beta}\right)\right\} j=1,2, \cdots m ; \beta \in X_{1}
\end{array}
$$

Where $G$ stands for geometric shape and dimensions, $P$ stands for position parameters, and $A$ stands for attributive information such as material prosperities, surfaces specifications or tolerances. $N$ stands 
for the normal vector of $f s$ to indicate the active face. $P R$ stands for performance requirements and the sum of $n_{j}$ equals $n$.

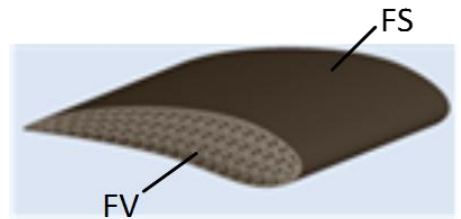

(a) Airefoil

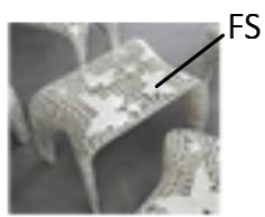

(b) aesthetic stool

Figure 6: Examples of functional surface and functional volume [23]

The detailed procedure to achieve function integration can be seen in Figure 7. Firstly, functional surfaces are extracted from the initial CAD model according to FRs. Other surfaces such as manufacturing surfaces and assembly surfaces are discarded. FSs in this stage has specific geometric information, dimensions, and spatial locations. Secondly, the extracted surfaces are compared and analyzed the possibility of surface-level function integration, otherwise, separated interface pairs are inserted. The last step is to link these FSs to form a solid entity to achieve part-level function integration.

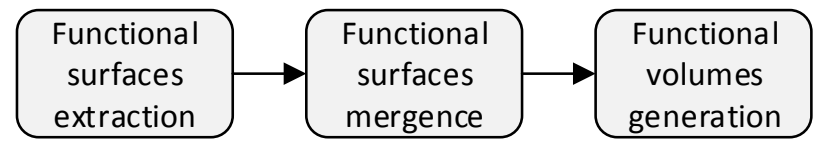

Figure 7: Procedure to achieve function integration

\subsection{Lattice Generation and Optimization}

In the design stage of lattice generation and optimization, to reduce the weight and cost of products, lattice structures on a meso-level is used to replace the solid material in chosen functional volumes generated in the functional integration stage. Then, a heterogeneous lattice structure designed method is proposed to optimize the strut thickness and lattice distribution to further optimize their functional performance. The detailed design flow of this design stage can be divided into four steps which are shown in Figure 8.

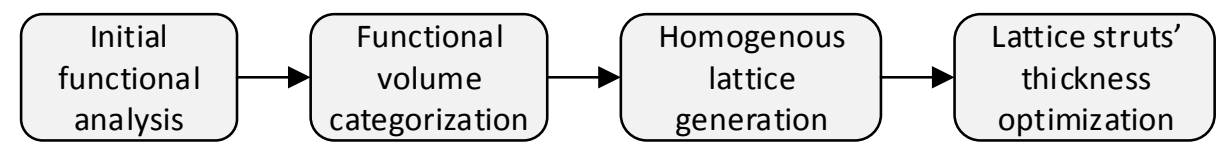

Figure 8: Design flow of lattice generation and optimization

In the first step, an initial functional analysis model is firstly built to calculate the structural response or performance of a product. For example, in the structure design field, Finite Element Analysis (FEA) model is usually regarded as functional analysis model to analyze the stress and displacement distribution of product with complex geometry. To reduce the computational load of the analysis model, functional volume is always filled with solid material whose properties are simple to calculate. After initial analysis, functional volumes are further categorized into two categories. The volumes whose stress is relatively high will be filled with solid material, and those functional volumes whose stress is relatively low will filled with lattice structure. Besides these two types of functional 
volumes, there will be the third type of functional volume whose stress only concentrates in a small region. This type of functional volume will be further divided into two sub-function volumes according to its stress distribution and macro-geometry. And these sub-functional volumes can be further categorized into the first two types. In the third step, the required properties of lattice structure on a meso-level can be calculated. The material chart and traditional material selection method can be used to select appropriate lattice topology and initial struts' thickness. Then based on these parameters, the initial designed product with homogenous lattice structure is generated.

To further improve the functional performance of product, lattice struts' thickness is optimized in the fourth step. In this step, the topology optimization based heterogeneous lattice structure design method is used to reduce the number of design parameters during the optimization process. In this design method, SIMP (Solid Isotropic Material with Penalization) method is firstly used to obtain the optimal relative density distribution in the functional volume of lattice structures. Then the mapping function $f$ is defined as:

$$
t=f\left(\rho^{*}\right) \quad \rho^{*} \in P, t \in T
$$

Where $\rho^{*}$ is the average relative density of elements in a certain lattice unit cell, $t$ is the struts' thickness of that lattice unit cell. This mapping function must satisfy the following two requirements:

(1) Suppose $\rho_{1}^{*}$ and $\rho_{2}^{*}$ are the average relative density for two different lattice unit cells, let $\rho_{1}^{*}<\rho_{2}^{*}$, then $f\left(\rho_{1}^{*}\right)<f\left(\rho_{2}^{*}\right)$;

(2) Mapping function $f$ is bijective from $P$ to $T$.

Based on this mapping function, the optimal thickness distribution of lattice structure is calculated. In order to further optimize this lattice structure, the lattice structures in the place whose relative density is smaller than a certain area is removed. After this step, an optimized heterogeneous lattice structure is obtained.

\section{Case study and discussion}

As shown in Figure 9 (a), an original triple clamp made in stainless steel is composed of 19 parts. Because of the assembly access requirements, part 1 and part 2 need to be fabricated separately and assembled. However, part 2, 3, and 4 are separate because of the consideration of manufacturing difficulties and cost. For example, the top surface of part 4 and that of part 3 are lined up with an angle of 10 degrees. There are two main disadvantages of this original design: excessive assembly operations and being heavy. With AM process, this part can be redesigned with the part consolidation method proposed in this paper. The redesign process is illustrated in Figure 9.

The first step is to define functional surfaces from the original CAD model and design specifications. As shown in step (b), functional surfaces are defined according to the FRS of providing support to external parts. These functional surfaces are colored in yellow. The second step is to extract these FSs that are described by exact information of position and surface boundaries as well as ambiguous information of surface thickness as shown in step (c). The third step is to check if these FSs can be merged together to achieve surface-level function integration. Since all these FSs are restricted by the fixed position for the need of external interfaces, this step cannot be conducted. The fourth step is to link all these FSs to form a solid and the linking volume is calculated as the minimum volume to envelop all these isolated FSs to achieve part-level function integration. The following step is to categorize the essential FVs which are filled with lattice structure according to topology analysis. The last step is to optimize the homogeneous structure's struts' thickness to generate homogeneous structure with skins. After all these steps, a triple clamp is redesigned with lattice structure and skins.

The redesigned part results in a lower mass of the blank part by approximately $20 \%$ and the part count is reduced from 19 to 7 . It allows for less assembly, less material consumption and better 
performance. However, an FEA analysis is needed to optimize the obtained functional volume to get a better result in the following research.

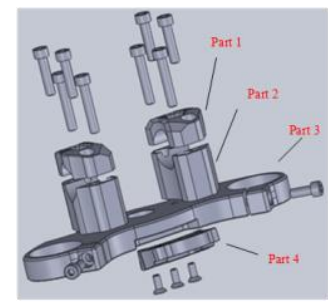

(a) exploded view

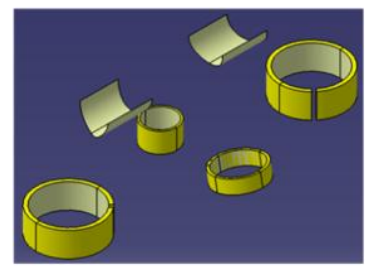

(c) functional surfaces extraction

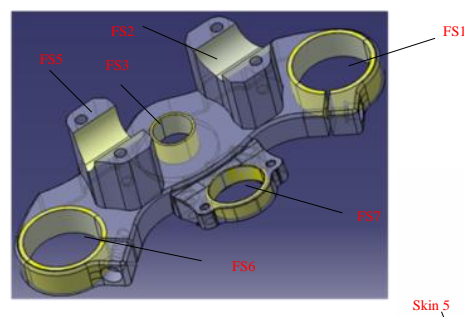

(b) define functional surfaces
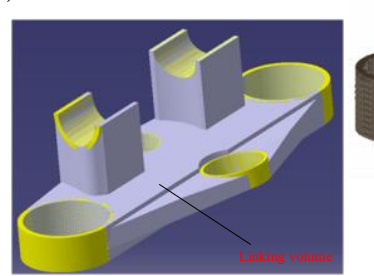

(e) homogeneous lattice structure and skin

Figure 9: The redesign process of a triple clamp.

\section{Conclusions and future research}

In this paper, conventional part consolidation methods and current AM enabled part consolidation methods are reviewed. The limitations of conventional part consolidation methods are classified into three categories. The first drawback is the extreme dependence on designers' experience and understanding of functional requirements. The second one is that there is no clear design framework of how to achieve less part count at design stage. The last one is that performance improvement is rarely involved. Therefore, an innovative part consolidation method focusing on function integration and performance improvement is proposed in this research. This design method consists of two steps. Firstly, achieve surface-level function integration and part-level function integration according to functional requirements. Secondly, functional volumes generated in the first step is categorized and optimized with heterogeneous lattice structures for the purpose of performance improvement. The case study proves that the proposed design method is effective in achieving less part count and better structure performance. To further improve this design method, more research work should be done.

(1) A design method from the upstream design phases should be developed. In this paper, the starting point is to optimize an existing part in order to find a locally optimal design solution with given design specifications.

(2) An analytic model for design process rationalization and multidisciplinary optimization is needed since most of the available design methods are semantic representation which is difficult to be implemented and to keep design flow consistent.

(3) A heterogeneous design method should be established to decide parameters of different struts in single lattice unit. For example, the strut along the direction of maximum normal stress should be thicker than other strut inside the lattice unit. 


\section{Acknowledge}

Financial support from the National Sciences and Engineering Research Council of Canada Discovery Grant RGPIN 436055-2013 and McGill Engineering Doctoral Award (MEDA) is acknowledged with gratitude.

\section{References}

[1] Encyclo, 2014, http://www.encyclo.co.uk/define/Part\%20consolidation.

[2] Andreasen, M. M., Kähler, S., and Lund, T., 1983, Design for assembly, Ifs Publications London.

[3] Crowther, P., 1999, "Design for disassembly," BDP Environment Design Guide.

[4] Boothroyd, G., Dewhurst, P., Knight, W. A., and Press, C., 2002, Product design for manufacture and assembly, M. Dekker New York.

[5] Hague, R., Mansour, S., and Saleh, N., 2003, "Design opportunities with rapid manufacturing," Assembly Automation, 23(4), pp. 346-356.

[6] Standard, A., "F2792. 2012. Standard Terminology for Additive Manufacturing Technologies," ASTM F2792-10e1.

[7] Hopkinson, N., and Dickens, P., 2006, "Emerging rapid manufacturing processes," Rapid manufacturing - an industrial revolution for the digital age. John Wiley, Chichester, pp. 55-80.

[8] Gibson, I., Rosen, D. W., and Stucker, B., 2010, Additive Manufacturing Technologies:Rapid Prototyping to Direct Digital Manufacturing.

[9] Prakash, W. N., Sridhar, V. G., and Annamalai, K., 2014, "New product development by DFMA and rapid prototyping," ARPN Journal of Engineering and Applied Sciences, 9(3), pp. 274-279.

[10] Hopkinson, N., Gao, Y., and McAfee, D. J., 2006, "Design for environment analyses applied to rapid manufacturing," Proceedings of the Institution of Mechanical Engineers Part D-Journal of Automobile Engineering, 220(D10), pp. 1363-1372.

[11] Becker, R., Grzesiak, A., and Henning, A., 2005, "Rethink assembly design," Assembly automation, 25(4), pp. 262-266.

[12] Ponche, R., Hascoet, J. Y., Kerbrat, O., and Mognol, P., 2012, "A new global approach to design for additive manufacturing," Virtual and Physical Prototyping, 7(2), pp. 93-105.

[13] Hao, L., and Raymond, D., "Design and additive manufacturing of cellular lattice structures," Proc. The International Conference on Advanced Research in Virtual and Rapid Prototyping (VRAP). Taylor \& Francis Group, Leiria, pp. 249-254.

[14] Chen, Y., 2007, "3d texture mapping for rapid manufacturing," Computer-Aided Design and Applications, 4(6), pp. 761-771.

[15] Wang, H., Chen, Y., and Rosen, D. W., "A hybrid geometric modeling method for large scale conformal cellular structures," Proc. ASME 2005 International Design Engineering Technical Conferences and Computers and Information in Engineering Conference, American Society of Mechanical Engineers, pp. 421-427.

[16] Ashby, M. F., and Cebon, D., 1993, "Materials selection in mechanical design," Le Journal de Physique IV, 3(C7), pp. C7-1-C7-9.

[17] Sundararajan, V. G., 2010, "Topology optimization for additive manufacturing of customized meso-structures using homogenization and parametric smoothing functions."

[18] Rezaie, R., Badrossamay, M., Ghaie, A., and Moosavi, H., 2013, "Topology optimization for fused deposition modeling process," Procedia CIRP, 6, pp. 521-526.

[19] Rosen, D. W., 2007, "Computer-aided design for additive manufacturing of cellular structures," Computer-Aided Design and Applications, 4(5), pp. 585-594.

[20] Wang, H. V., 2005, "A unit cell approach for lightweight structure and compliant mechanism," Citeseer. 
[21] Tang, Y., and Zhao, Y. F., 2014, "Design Method for Lattice-Skin Structure Fabricated by Additive Manufacturing," ASME 2014 International Mechanical Engineering Congress \& Exposition,Montréal, Canada.

[22] Dieter, G. E., Schmidt, L. C., and AZARM, S., 2009, "Engineering design," American Society of Mechanical Engineers.

[23] Bin Maidin, S., Campbell, I., and Pei, E., 2012, "Development of a design feature database to support design for additive manufacturing," Assembly Automation, 32(3), pp. 235-244. 